\title{
Monogamy inequality for any local quantum resource and entanglement
}

\author{
S. Camalet \\ Laboratoire de Physique Théorique de la Matière Condensée, UMR 7600, \\ Sorbonne Universités, UPMC Univ Paris 06, F-75005, Paris, France
}

\begin{abstract}
We derive a monogamy inequality for any local quantum resource and entanglement. It results from the fact that there is always a convex measure for a quantum resource, as shown here, and from the relation between entanglement and local entropy. One of its consequences is an entanglement monogamy different from that usually discussed. If the local resource is nonuniformity or coherence, it is satisfied by familiar resource and entanglement measures. The ensuing upper bound for the local coherence, determined by the entanglement, is independent of the basis used to define the coherence.
\end{abstract}

The more a two-level system is quantum-mechanically entangled with another two-level system, the less it can be entangled with a third one [1]. This behavior, known as entanglement monogamy, has also been found for larger systems [2 4]. Quantitatively, it is described by an inequality, involving a bipartite entanglement monotone. The term "monotone" refers to the fact that a proper measure of entanglement, cannot increase under specific transformations of quantum states. They are those that can be achieved by local operations and classical communication, and hence, cannot generate entanglement $5-7$.

Quantum entanglement theory is a quantum resource theory. This approach to quantum resources, is based on the definition of free states, for which the resource vanishes, and free operations. The set of these allowed operations, depends on the considered theory [8]. But, in any case, they transform free states into free states [9]. For entanglement, the free states are the socalled separable states [10], and the free operations are those obtained from local operations and classical communication. Other examples of quantum resources are nonuniformity [11 13], athermality [14 17], and coherence [8, 18 20]. Contrary to entanglement, the definitions of these resources, do not rely on a partition of the system of interest. Similarly to entanglement monotones, a measure of a given resource, cannot increase under the corresponding free operations.

Recently, a monogamy inequality for entanglement and local contextuality, has been derived [21, 22]. It shows that the entanglement between two systems, and the violation, by one of them, of a state-dependent noncontextuality inequality, constrain each other. In the context of open sytems, the development of the entanglement between a system and its environment, due to their mutual interaction, is commonly seen as playing an essential role in the decoherence of the system 23, 24]. These influences on the quantum properties of a system, of its entanglement with another system, suggest that there may be a general monogamy relation between any local quantum resource and entanglement. The interplay between entanglement and local coherence, has also been discussed from other perspectives [19, 20].

In this Letter, we derive a monogamy inequality for any local quantum resource and entanglement. This inequality involves a convex measure of the resource. Though, for resource measures, convexity is frequently assumed [18], it is not a basic axiom [7, 19]. However, we show that, as soon as there exists a proper measure for a resource, i.e., that does not increase under free operations, there is also a convex one. The derivation of the monogamy inequality, essentially relies on the relation between entanglement and local entropy 22]. By entropy, we mean a non-negative function of quantum states, that depends only on the state eigenvalues, is nondecreasing with the state mixedness, in the sense of majorization [25], and vanishes for pure states [26, 27]. The found monogamy inequality is discussed for three local resources: entanglement, nonuniformity, and coherence.

Two essential requirements for a resource measure, are that (i) it is non-negative and vanishes for free states, (ii) it is non-increasing under free operations. Such a measure consists of a set of functions $R_{d}$, from $d \times d$ density matrices to real numbers [13], or, more generally, of a set of functions $R_{\boldsymbol{d}}$, where $\boldsymbol{d} \equiv\left(d_{1}, d_{2}, \ldots\right)$, from density matrices on the Hilbert space $\mathcal{H}_{d_{1}} \otimes \mathcal{H}_{d_{2}} \otimes \ldots$, to real numbers. Condition (i) simply means that $R_{\boldsymbol{d}} \geq 0$, and $R_{\boldsymbol{d}}\left(\rho_{\mathrm{A}}\right)=0$ when $\rho_{\mathrm{A}}$ is free, for any $\boldsymbol{d}$. We denote $\rho_{\mathrm{A}}$ the state of the system of interest, possibly composite, named A, since a bipartite system, consisting of A and another system, is considered in the following. Note that $R_{d}$ can vanish for some resourceful states. The important point is that it is zero for all free states [7, 18]. The monotonicity condition (ii) reads, more precisely, as

$$
R_{\boldsymbol{d}^{\prime}}\left(\sum_{q} K_{q} \rho_{\mathrm{A}} K_{q}^{\dagger}\right) \leq R_{\boldsymbol{d}}\left(\rho_{\mathrm{A}}\right),
$$

where $K_{q}$ are the Kraus operators of the considered free operation, which are such that $\sum_{q} K_{q}^{\dagger} K_{q}$ is equal to the identity operator on $\mathcal{H}_{\boldsymbol{d}} \equiv \mathcal{H}_{d_{1}} \otimes \mathcal{H}_{d_{2}} \otimes \ldots$. It can also be understood as

$$
\sum_{q} p_{q} R_{\boldsymbol{d}_{q}}\left(\rho_{\mathrm{A}}^{(q)}\right) \leq R_{\boldsymbol{d}}\left(\rho_{\mathrm{A}}\right)
$$

where $p_{q}=\operatorname{tr}\left(K_{q}^{\dagger} K_{q} \rho_{\mathrm{A}}\right)$ is the probability of outcome $q$, $\rho_{\mathrm{A}}^{(q)}=K_{q} \rho_{\mathrm{A}} K_{q}^{\dagger} / p_{q}$ is the corresponding state, and the 
sum runs over $q$ such that $p_{q}>0$ [18]. In eq.(11), all the linear maps $K_{q}$ are from $\mathcal{H}_{\boldsymbol{d}}$ to $\mathcal{H}_{\boldsymbol{d}^{\prime}}$, with the same $\boldsymbol{d}^{\prime}$, whereas, in eq.(2), the vectors $\boldsymbol{d}_{q}$ can be different from one another. As shown in the supplemental material 28], if there are functions $R_{\boldsymbol{d}}$ satisfying the above points (i) and (ii), then there are convex ones, $R_{d}^{c h}$, which obey the same conditions. They fulfill (ii) in the same way as the set $\left\{R_{\boldsymbol{d}}\right\}$ does. Besides, $\left\{R_{d}^{c h}\right\}$ always obeys eq.(1), since the functions $R_{d}^{c h}$ are convex [18]. As $R_{d}^{c h} \leq R_{d}$, see supplemental material, it vanishes whenever $R_{\boldsymbol{d}}$ does, but it can be zero for other states. If, for example, the set of free states is not convex, there are resourceful states which are convex combinations of free states. For these states, $R_{d}^{c h}$ necessarily vanishes, but $R_{d}$ may not.

Let us now consider an arbitrary convex function $R_{\boldsymbol{d}}$, of the density operators on $\mathcal{H}_{\boldsymbol{d}}$, non-negative and bounded. From $R_{\boldsymbol{d}}$, we define, for any state $\rho_{\mathrm{A}}$ of any finite system $\mathrm{A}$,

$$
G\left(\rho_{\mathrm{A}}\right) \equiv \sup _{\{|i\rangle\}} R_{\boldsymbol{d}}\left(\sum_{i=1}^{r} \lambda_{i}\left(\rho_{\mathrm{A}}\right)|i\rangle\langle i|\right),
$$

for $r \leq n(\boldsymbol{d})$, and 0 otherwise, where $n(\boldsymbol{d}) \equiv d_{1} d_{2} \ldots$ is the dimension of $\mathcal{H}_{\boldsymbol{d}}, r$ is the rank of $\rho_{\mathrm{A}}$, and $\lambda_{i}(M)$ denotes the eigenvalues of the Hermitian operator $M$, with $\lambda_{i}(M) \geq \lambda_{i+1}(M)$. The supremum is taken over the bases $\{|i\rangle\}$ of $\mathcal{H}_{\boldsymbol{d}}$. The function (3) depends on $\rho_{\mathrm{A}}$ only via the nonvanishing eigenvalues $\lambda_{i}\left(\rho_{\mathrm{A}}\right)$. Contrary to $\boldsymbol{R}_{\boldsymbol{d}}$, it is defined for any state, and does not depend on the corresponding Hilbert space. To make this distinction clear, we do not denote its dependence on $\boldsymbol{d}$, which simply comes from the definition (3). If the Hilbert space of A is $\mathcal{H}_{\boldsymbol{d}}$, eq.(3) reduces to $G\left(\rho_{\mathrm{A}}\right)=\sup _{U} R_{\boldsymbol{d}}\left(U \rho_{\mathrm{A}} U^{\dagger}\right)$, where the supremum is taken over the unitary operators $U$ of $\mathcal{H}_{\boldsymbol{d}}$, and hence, $R_{\boldsymbol{d}}\left(\rho_{\mathrm{A}}\right) \leq G\left(\rho_{\mathrm{A}}\right)$.

It follows, from the properties of $\mathrm{G}$, shown in the supplemental material, that $R_{\boldsymbol{d}}^{\text {sup }} \equiv G(|i\rangle\langle i|)$, where $|i\rangle$ is any pure state, is the supremum of $R_{\boldsymbol{d}}$, and that the function $S$, defined by

$$
S\left(\rho_{\mathrm{A}}\right) \equiv R_{\boldsymbol{d}}^{\text {sup }}-G\left(\rho_{\mathrm{A}}\right),
$$

is non-negative. Furthermore, $-S$ is Schur-convex, and, by construction, $S$ vanishes when $\rho_{\mathrm{A}}$ is pure. Thus, $S$ is an entropy, and can obey, with an entanglement monotone $E$,

$$
S\left(\rho_{\mathrm{A}}\right)=\max _{\rho: \operatorname{tr}_{\mathrm{B}} \rho=\rho_{\mathrm{A}}} E(\rho),
$$

where the maximum is taken over the states $\rho$ of the composite systems, consisting of $\mathrm{A}$, and another system, say $\mathrm{B}$, such that $\operatorname{tr}_{\mathrm{B}} \rho=\rho_{\mathrm{A}}$, and $\operatorname{tr}_{\mathrm{B}}$ denotes the partial trace over $B$ [22]. The maximum is reached for pure states $\rho$. Note that an entanglement monotone does not depend explicitly on the Hilbert space dimensions of A an B [7, 31]. Since the function (4) is concave, an explicit entanglement monotone, that fulfills eq.(5), with $S$, can be built. It is the convex roof

$$
E^{c r}(\rho) \equiv \inf _{\left\{P_{k},\left|\Psi_{k}\right\rangle\right\}} \sum_{k} P_{k} S\left(\operatorname{tr}_{\mathrm{B}}\left|\Psi_{k}\right\rangle\left\langle\Psi_{k}\right|\right),
$$

where the infinum is taken over the ensembles $\left\{P_{k},\left|\Psi_{k}\right\rangle\right\}$ such that $\sum_{k} P_{k}\left|\Psi_{k}\right\rangle\left\langle\Psi_{k}\right|=\rho[5,22]$. It is clearly lower than $S\left(\rho_{\mathrm{A}}\right)$, as $S$ is concave. Expression (6) with $S$ replaced by the von Neumann entropy, is the definition of the entanglement of formation [7].

Equation (5) gives the monogamy inequality

$$
R_{\boldsymbol{d}}\left(\rho_{\mathrm{A}}\right)+E(\rho) \leq R_{\boldsymbol{d}}^{\text {sup }},
$$

when the Hilbert space of system $\mathrm{A}$ is $\mathcal{H}_{\boldsymbol{d}}$. The entanglement monotone (6) satisfies this inequality, but it may not be the only one. As soon as a monotone $E$ obeys eq.(5) with $S$, it fulfills eq.(7). Moreover, for such an entanglement measure, there are, for any local eigenspectrum $\left\{\lambda_{i}\left(\rho_{\mathrm{A}}\right)\right\}_{i=1}^{n(\boldsymbol{d})}$, global states $\rho$ such that the left side of eq.(17), is as close as we wish to $R_{d}^{\text {sup }}$, as shown in the supplemental material. As mentioned above, for any quantum resource, there is a convex measure of it, and hence, a monogamy inequality (7) for the entanglement between $\mathrm{A}$ and $\mathrm{B}$, and this resource for A. For the set of states $\rho$ such that the two sides of eq.(17) are equal, or infinitely close to each other, an increase of the entanglement $E(\rho)$, means a reduction of the local resource $R_{\boldsymbol{d}}\left(\rho_{\mathrm{A}}\right)$, of the same amount, and reciprocally. In general, entanglement and local resource limit each other. We remark that the monogamy inequality for entanglement and local contextuality, derived in Ref. 22], is a particular case of eq.(7), with $R_{\boldsymbol{d}}$ replaced by the convex function $C_{d}$ 21].

The entanglement between $\mathrm{A}$ and $\mathrm{B}$, is not changed by a unitary transformation $U$ performed on $\mathrm{A}$, and hence, $\rho_{\mathrm{A}}$ can be replaced by $U \rho_{\mathrm{A}} U^{\dagger}$, in eq.(17). Thus, $R_{\boldsymbol{d}}^{\text {sup }}-E(\rho)$ not only upperbounds $R_{\boldsymbol{d}}\left(\rho_{\mathrm{A}}\right)$, but also all the values of $R_{\boldsymbol{d}}$ that can be obtained by performing local unitary transformations on A. This bound can be reached, in this way, when $\rho$ is pure. For the entanglement between $\mathrm{A}$ and $\mathrm{B}$, inequality (7) gives an upper bound, $R_{\boldsymbol{d}}^{\text {sup }}-\sup _{U} R_{\boldsymbol{d}}\left(U \rho_{\mathrm{A}} U^{\dagger}\right)$, where the supremum is taken over the unitary operators $U$ of $\mathrm{A}$, that depends only on the eigenvalues of the local state $\rho_{\mathrm{A}}$. We remark that the supremum of $R_{\boldsymbol{d}}$, was obtained above as $R_{\boldsymbol{d}}^{\text {sup }}=\sup _{|i\rangle} R_{\boldsymbol{d}}(|i\rangle\langle i|)$, where the supremum is taken over the pure states $|i\rangle$ of $\mathcal{H}_{\boldsymbol{d}}$. The convexity of $R_{\boldsymbol{d}}$, implies thus that it is equal, or infinitely close to its supremum, for some pure states. However, the above results do not impose that there exist such states independent of the resource measure. As is well known, this is the case for entanglement and coherence [7, 18].

An interesting particular case is when the system A is made up of two subsystems, $A_{1}$ and $A_{2}$, and the considered resource for $A$, is the entanglement between $A_{1}$ 
and $\mathrm{A}_{2}$. Then, inequality (7) can be rewritten, in a more familiar form, as

$$
\tilde{E}\left(\mathrm{~A}_{1}: \mathrm{A}_{2}\right)+E\left(\mathrm{~A}_{1} \mathrm{~A}_{2}: B\right) \leq \tilde{E}_{\max }
$$

where the entanglement $\tilde{E}\left(\mathrm{~A}_{1}: \mathrm{A}_{2}\right)$ between $\mathrm{A}_{1}$ and $\mathrm{A}_{2}$, and the entanglement $E\left(\mathrm{~A}_{1} \mathrm{~A}_{2}: B\right)$ between $\mathrm{A}$ and $\mathrm{B}$, are evaluated for the common state $\rho$ of $\mathrm{A}_{1}, \mathrm{~A}_{2}$, and $\mathrm{B}$. The right side, $\tilde{E}_{\text {max }}$, is the maximum value of $\tilde{E}\left(\mathrm{~A}_{1}: \mathrm{A}_{2}\right)$, reached when $\rho_{\mathrm{A}}=\operatorname{tr}_{\mathrm{B}} \rho$ is a maximally entangled state of $\mathrm{A}_{1}$ and $\mathrm{A}_{2}$. Note that $E\left(\mathrm{~A}_{1} \mathrm{~A}_{2}: B\right)$ is also bounded by $\tilde{E}_{\max }$. It attains this value for pure states $\rho=|\psi\rangle\langle\psi|$, where $|\psi\rangle$ has Schmidt coefficients such that $\rho_{\mathrm{A}}$ is absolutely separable [32, 33]. The entanglement monogamy described by inequality (8), is different from that usually discussed [1-4, 7]. Equation (8) shows that the entanglement between two parts of a system, and the entanglement of this system with another one, limit each other. In the extreme case, usually used to illustrate entanglement monogamy, of two maximally entangled systems $A_{1}$ and $\mathrm{A}_{2}$, it gives $E\left(\mathrm{~A}_{1} \mathrm{~A}_{2}: B\right)=0$, as expected, since system $\mathrm{A}$ is in a pure state, and hence not correlated to any other one.

An inequality, similar to eq.(8), but involving only one entanglement monotone, the negativity $E_{\mathcal{N}}$ 7, 34, 35], can be obtained. Any measure of the form (6), is larger than $g\left[E_{\mathcal{N}}(\rho)\right]$, where $g$ is a non-decreasing function, given by $g(x)=c o(h)(2 x+1)$, with $c o(h)$ the convex hull of $h$, defined, on $\left[1, d^{*}\right]$, by $h(y) \equiv$ $\inf _{\left\{p_{i}\right\} \in \mathcal{F}(y)} S\left(\sum_{i=1}^{d^{*}} p_{i}|i\rangle\langle i|\right)$. In this expression, $d^{*}$ is the smallest of the Hilbert space dimensions of $\mathrm{A}$ and $\mathrm{B},\{|i\rangle\}_{i=1}^{d^{*}}$ is an arbitrary basis, and $\mathcal{F}(y)$ is the set of $\left\{p_{i}\right\}_{i=1}^{d^{*}}$ such that $p_{i} \geq 0, \sum_{i=1}^{d^{*}} p_{i}=1$, and $\sum_{i=1}^{d^{*}} \sqrt{p_{i}}=$ $\sqrt{y}$ [22, 36]. Thus, for $\tilde{E}=E_{\mathcal{N}}$, eq. (8) leads to $E_{\mathcal{N}}\left(\mathrm{A}_{1}\right.$ : $\left.\mathrm{A}_{2}\right)+g\left[E_{\mathcal{N}}\left(\mathrm{A}_{1} \mathrm{~A}_{2}: B\right)\right] \leq E_{\mathcal{N}, \text { max }}$, where $E_{\mathcal{N}, \text { max }}$ is the maximum value of $E_{\mathcal{N}}\left(\mathrm{A}_{1}: \mathrm{A}_{2}\right)$. The second term on the left side can also reach this value. This can be seen as follows. For a maximally entangled state of A and $\mathrm{B}, E_{\mathcal{N}}(\mathrm{A}: \mathrm{B})=\left(d^{*}-1\right) / 2$ [35]. Since $h$, and its convex hull, are defined on the finite interval $\left[1, d^{*}\right]$, $g\left(d^{*} / 2-1 / 2\right)=h\left(d^{*}\right)$. The only element of $\mathcal{F}\left(d^{*}\right)$ is $p_{i}=1 / d^{*}$. Thus, if the Hilbert space dimension of $\mathrm{A}$ is not larger than that of $\mathrm{B}, h\left(d^{*}\right)$ is determined by the maximally mixed state of $\mathrm{A}$, which is invariant under unitary transformations, and not entangled, and hence, using eq.(4), $h\left(d^{*}\right)=E_{\mathcal{N}, \max }$. The function $g$ defined above, depends on the systems considered. For a system A consisting of two two-level systems, it can be evaluated explicitly, see supplemental material.

Inequality (8) can be generalised to more than three systems. The usual inequality for entanglement monogamy, for $N$ systems $\mathrm{A}_{k}$, reads

$$
\sum_{k \geq 2} \tilde{E}\left(\mathrm{~A}_{1}: \mathrm{A}_{k}\right) \leq \tilde{E}\left(\mathrm{~A}_{1}: \mathrm{A}_{2} \ldots \mathrm{A}_{N}\right) .
$$

It has been derived for two-level systems and different entanglement monotones [1, 3 , 位], and for systems of any sizes and squashed entanglement [2]. For a system A consisting of $N$ subsystems, inequalities (8) and (9) lead directly to

$$
\sum_{k \geq 2} \tilde{E}\left(\mathrm{~A}_{1}: \mathrm{A}_{k}\right)+E\left(\mathrm{~A}_{1} \ldots \mathrm{A}_{N}: B\right) \leq \tilde{E}_{\max }
$$

which relates the entanglement of one subsystem of A, with each of the others, and the entanglement between $\mathrm{A}$ and B. Other inequalities, for the entanglement between parts of a system, and its entanglement with another system, can be derived from eq.(8) and eq.(9). For example, for three subsystems, they give

$$
\frac{2}{3} \sum_{1 \leq k<l \leq 3} \tilde{E}\left(\mathrm{~A}_{k}: \mathrm{A}_{l}\right)+E\left(\mathrm{~A}_{1} \mathrm{~A}_{2} \mathrm{~A}_{3}: B\right) \leq \tilde{E}_{\max } .
$$

To compare inequalities (8) and (9), we consider three identical systems $\mathrm{A}_{k}$ in a permutation-symmetric state. For such a state, the entanglement between any two systems, is equal to that between $A_{1}$ and $A_{2}$, and the entanglement between a system and the two other ones, is equal to that between $A_{1}$ and the composite system $\mathrm{A}_{2} \mathrm{~A}_{3}$, consisting of $\mathrm{A}_{2}$ and $\mathrm{A}_{3}$. Inequalities (8) and (9) yield, respectively, $\tilde{E}\left(\mathrm{~A}_{1}: \mathrm{A}_{2}\right) \leq \tilde{E}_{\max }-E\left(\mathrm{~A}_{1}: \mathrm{A}_{2} \mathrm{~A}_{3}\right)$, and $\tilde{E}\left(\mathrm{~A}_{1}: \mathrm{A}_{2}\right) \leq \tilde{E}\left(\mathrm{~A}_{1}: \mathrm{A}_{2} \mathrm{~A}_{3}\right) / 2$. The above first inequality shows that the two-system entanglement, and the entanglement of the bipartitions of the global system, constrain each other, but the second one does not. Assume, for instance, the state of the global system is pure. In this case, $\tilde{E}\left(\mathrm{~A}_{1}: \mathrm{A}_{2} \mathrm{~A}_{3}\right)$, and $E\left(\mathrm{~A}_{1}: \mathrm{A}_{2} \mathrm{~A}_{3}\right)$, are given by the corresponding entropies, evaluated for $\rho_{\mathrm{A}_{1}}$. As this state depends only on one probability, $E\left(\mathrm{~A}_{1}: \mathrm{A}_{2} \mathrm{~A}_{3}\right)$, for $\tilde{E}=E_{\mathcal{N}}$, can be written in terms of $E_{\mathcal{N}}\left(\mathrm{A}_{1}: \mathrm{A}_{2} \mathrm{~A}_{3}\right)$, using the results of Ref.[33]. Moreover, for three twolevel systems in a pure state, inequality (9) holds for $E_{\mathcal{N}}^{2}$ 37]. Finally, we find

$$
E_{1} \leq \min \left\{\sqrt{2} E_{2}, \sqrt{1-2 E_{2}^{2}}+\sqrt{1 / 4-E_{2}^{2}}-1 / 2\right\} / 2,
$$

where $E_{1}=E_{\mathcal{N}}\left(\mathrm{A}_{1}: \mathrm{A}_{2}\right)$, and $E_{2}=E_{\mathcal{N}}\left(\mathrm{A}_{1}: \mathrm{A}_{2} \mathrm{~A}_{3}\right)$. The first term on the right side, comes from eq.(9), and increases with $E_{2}$, whereas the second one comes from eq.(8), and decreases with $E_{2}$. They are equal for $E_{2} \simeq$ 0.415 . Thus, for $E_{2} \geq 0.416$, the relation between $E_{1}$ and $E_{2}$, is better described by inequality (8). With the entanglement of formation in place of $E_{\mathcal{N}}$, the situation is similar, but $E\left(\mathrm{~A}_{1}: \mathrm{A}_{2} \mathrm{~A}_{3}\right)$ cannot be expressed explicitly.

As a second example of quantum resource, we consider nonuniformity. From the von Neumann entropy $S_{v N}$, a measure $R_{d}$ of this resource, can be defined by $R_{d}\left(\rho_{\mathrm{A}}\right)=\log d-S_{v N}\left(\rho_{\mathrm{A}}\right)$, where $d$ is the Hilbert space dimension of A [13]. The same expression with a Rényi entropy $S_{R}$, of positive order, in place of $S_{v N}$, gives also a nonuniformity monotone 13. Such measures depend on $\rho_{\mathrm{A}}$ only via the eigenvalues $\lambda_{i}\left(\rho_{\mathrm{A}}\right)$. Thus, eq.(3) and eq.(44) yield $S\left(\rho_{\mathrm{A}}\right)=S_{v N}\left(\rho_{\mathrm{A}}\right)$ if the rank of $\rho_{\mathrm{A}}$ is 
not greater than $d$, and $S\left(\rho_{\mathrm{A}}\right)=\log d$ otherwise, and similarly for $S_{R}$. However, here, entanglement monotones that fulfill eq.(5) with $S_{v N}$ or $S_{R}$, instead of $S$, are more useful. Such an entanglement measure $E$ satisfies the monogamy inequality (7) with $R_{d}$, for any value of $d$. This directly follows from $E(\rho) \leq S_{v N / R}\left(\rho_{\mathrm{A}}\right)$ and $R_{d}^{\text {sup }}=\log d$. Well-known entanglement monotones obey eq.(5) with $S_{v N}$, namely, distillable entanglement, entanglement cost, entanglement of formation, and relative entropy of entanglement [7, 38, 39]. Note that $-S_{R}$ is not necessarily convex, depending on its order, but is always Schur-convex [27], and can thus satisfy eq.(5). For a Tsallis entropy $S_{T}$, of positive order $q$, the procedure followed in ref.[13], to construct nonuniformity measures, leads to $R_{d}\left(\rho_{\mathrm{A}}\right)=R_{d}^{\text {sup }}-d^{q-1} S_{T}\left(\rho_{\mathrm{A}}\right)$, where $R_{d}^{\text {sup }}=\left(d^{q-1}-1\right) /(q-1)$. An entanglement monotone $E$ that fulfills eq.(5) with $S_{T}$, obeys, for any $d$, $R_{d}\left(\rho_{\mathrm{A}}\right)+d^{q-1} E(\rho) \leq R_{d}^{\text {sup }}$, which is also a monogamy inequality for entanglement and local nonuniformity.

We now turn to quantum coherence, for which the free states are the incoherent states, that are defined with respect to a specific basis $\{|i\rangle\}$ of the considered Hilbert space $\mathcal{H}_{d}$. A particularly interesting coherence measure is the relative entropy of coherence, which can be cast into the form $R_{d}\left(\rho_{\mathrm{A}}\right)=-\sum_{i=1}^{d} p_{i} \log p_{i}-S_{v N}\left(\rho_{\mathrm{A}}\right)$, where $p_{i}=\left\langle i\left|\rho_{\mathrm{A}}\right| i\right\rangle[18]$. It is clearly lower than $\log d-S_{v N}\left(\rho_{\mathrm{A}}\right)$. For a given density matrix $\rho_{\mathrm{A}}$, this value can be reached by performing unitary transformations [40], and hence $R_{d}^{\text {sup }}=\log d$. The situation is thus similar to that of the nonuniformity measure, based on $S_{v N}$, discussed above. So, $R_{d}$ obeys inequality (7), for any value of $d$, with familiar entanglement monotones. All the coherence monotones built with the help of a contractive distance [18], e.g., the relative entropy of coherence, satisfy $R_{d}\left(\{U|i\rangle\}, U \rho_{\mathrm{A}} U^{\dagger}\right)=R_{d}\left(\{|i\rangle\}, \rho_{\mathrm{A}}\right)$, for any unitary operator $U$, where we have denoted explicitly the dependence on the basis, with respect to which the incoherent states are defined. For such measures, the function $S$, given by eq.(44) and eq.(3), and the supremum $R_{d}^{\text {sup }}$, do not depend on the basis $\{|i\rangle\}$. This can be valid also for an entanglement monotone obeying eq.(5) with $S$. An example is given by the definition (6). In this case, the upper bound, $R_{d}^{\text {sup }}-E(\rho)$, to the coherence $R_{d}\left(\{|i\rangle\}, \rho_{\mathrm{A}}\right)$, is independent of $\{|i\rangle\}$. Moreover, for a pure state $\rho$, this bound is reached for some bases.

Using the above results, the role played by the entanglement of a system with its environment, in its decoherence, can be clarified. The coherence $R_{d}$ may vanish at long times, for a particular basis $\{|i\rangle\}$, whereas the entanglement with the environment, allows nonzero coherence for other bases. Consider, for instance, that A is a twolevel system, which interacts with a large system, B. For a pure dephasing Hamiltonian, and if $\mathrm{A}$ and $\mathrm{B}$ are initially in pure states, their common pure state reads $|\psi\rangle=$ $\sqrt{p}|0\rangle|\tilde{0}\rangle+\sqrt{1-p}|1\rangle|\tilde{1}\rangle$, where $p \in[0,1],\{|0\rangle,|1\rangle\}$ is a basis of $\mathcal{H}_{2}$, and the states $|\tilde{\imath}\rangle$ are such that $|\tilde{0}\rangle=|\tilde{1}\rangle$ at ini-

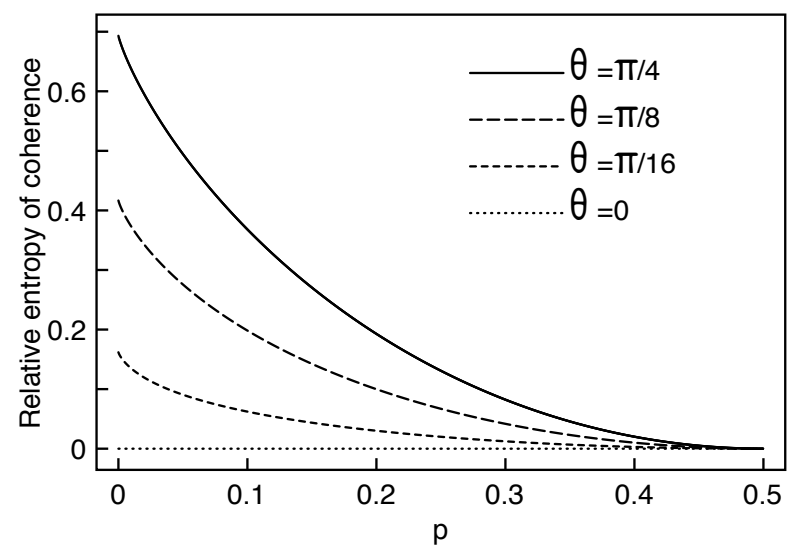

FIG. 1: Relative entropy of coherence for the long-time local state $p|0\rangle\langle 0|+(1-p)| 1\rangle\langle 1|$, discussed in the text, and the basis $\{\cos \theta|0\rangle+\sin \theta|1\rangle, \sin \theta|0\rangle-\cos \theta|1\rangle\}$, as a function of $p$, for four values of $\theta$. The coherence for $\theta=\pi / 4$, is $\log 2-h(p)$, where $h(p)$ is the entanglement of formation of the global state. It is the bound given by eq.(7).

tial time, and $\langle\tilde{0} \mid \tilde{1}\rangle$ goes to zero at long times [24, 41]. In this long time regime, the relative entropy of coherence, for the basis $\{|0\rangle,|1\rangle\}$, and the state $\rho_{\mathrm{A}}=\operatorname{tr}_{\mathrm{B}}|\psi\rangle\langle\psi|$, vanishes, and the entanglement of formation, for $\rho=|\psi\rangle\langle\psi|$, reaches $h(p) \equiv-p \log p-(1-p) \log (1-p)$. Inequality (7) implies only that the relative entropy of coherence cannot exceed $\log 2-h(p)$, which is not zero if $p \neq 1 / 2$. Since $\rho$ is pure, this bound is attained for some bases, e.g., $\{(|0\rangle+|1\rangle) / \sqrt{2},(|0\rangle-|1\rangle) / \sqrt{2}\}$. Figure 1 shows the relative entropy of coherence for $\rho_{\mathrm{A}}$, and different bases.

In summary, we have derived a monogamy inequality for any local quantum resource and entanglement. We have shown that there is always a convex measure for a quantum resource, and that, for such a measure, there is a concave entropy, which satisfies a simple inequality with it. The monogamy inequality then ensues from the existence, for any concave entropy, of a bipartite entanglement monotone, for which the entanglement of the global state is necessarily lower than the entropies of the local states 22]. This inequality has been discussed for three local resources. It shows that the entanglement between parts of a system, and the entanglement between this system and another one, constrain each other. This entanglement monogamy is different from that usually considered [1 4]. As seen, for three two-level systems in a pure symmetric state, this difference is manifest. For nonuniformity and coherence, the inequality can be written in terms of known resource measures [13, 18], and entanglement monotones, such as the entanglement of formation [7]. For a large class of coherence monotones, to which belong the familiar ones [18], it gives an upper bound to the local coherence, which is independent of the basis with respect to which the coherence is evaluated. This bound is reached for some bases, when the global 
state is pure. Due to its generality, we expect the found monogamy inequality to have other consequences, for the quantum resources considered here, or for other ones.

[1] V. Coffman, J. Kundu, and W.K. Wootters, Distributed entanglement, Phys. Rev. A 61, 052306 (2000).

[2] M. Koashi, and A.Winter, Monogamy of quantum entanglement and other correlations, Phys. Rev. A 69, 022309 (2004).

[3] T.J. Osborne, and F. Verstraete, General monogamy inequality for bipartite qubit entanglement, Phys. Rev. Lett. 96, 220503 (2006).

[4] Y.-K. Bai, Y.-F. Xu, and Z.D. Wang, General Monogamy Relation for the Entanglement of Formation in Multi-qubit Systems, Phys. Rev. Lett. 113, 100503 (2014).

[5] G. Vidal, Entanglement monotones, J. Mod. Opt. 47, 355 (2000).

[6] M. B. Plenio, and S. Virmani, An introduction to entanglement measures, Quant. Inf. Comput. 7, 1 (2007).

[7] R. Horodecki, P. Horodecki, M. Horodecki, and K. Horodecki, Quantum entanglement, Rev. Mod. Phys. 81, 865 (2009).

[8] E. Chitambar, and G. Gour, Critical Examination of Incoherent Operations and a Physically Consistent Resource Theory of Quantum Coherence, Phys. Rev. Lett. 117, 030401 (2016).

[9] F. G.S.L Brandão, and G. Gour, Reversible Framework for Quantum Resource Theories, Phys. Rev. Lett. 115, 070503 (2015).

[10] R.F. Werner, Quantum states with Einstein-PodolskyRosen correlations admitting a hidden-variable model, Phys. Rev. A 40, 4277 (1989).

[11] M. Horodecki, K. Horodecki, P. Horodecki, R. Horodecki, J. Oppenheim, A. Sen(De), and U. Sen, Local Information as a Resource in Distributed Quantum Systems, Phys. Rev. Lett. 90, 100402 (2003).

[12] M. Horodecki, P. Horodecki, and J. Oppenheim, Reversible transformations from pure to mixed states and the unique measure of information, Phys. Rev. A 67, 062104 (2003).

[13] G. Gour, M. P. Müller, V. Narasimhachar, R. W. Spekkens, and N. Y. Halpern, The resource theory of informational nonequilibrium in thermodynamics, Phys. Rep. 583, 1 (2015).

[14] D. Janzing, P. Wocjan, R. Zeier, R. Geiss, and T. Beth, Thermodynamic cost of reliability and low temperatures: Tightening Landauer's principle and the second law, Int. J. Theor. Phys. 39, 2717 (2000).

[15] J. Aberg, Truly work-like extraction via a single-shot analysis, Nat. Commun. 4, 1925 (2013).

[16] M. Horodecki and J. Oppenheim, Fundamental limitations for quantum and nanoscale thermodynamics, Nat. Commun. 4, 2059 (2013).

[17] F. G. S. L. Brandão, M. Horodecki, J. Oppenheim, J. M. Renes, and R. W. Spekkens, Resource Theory of Quantum States Out of Thermal Equilibrium, Phys. Rev. Lett. 111, 250404 (2013).

[18] T. Baumgratz, M. Cramer, and M.B. Plenio, Quantifying Coherence, Phys. Rev. Lett. 113, 140401 (2014).

[19] A. Streltsov, U. Singh, H.S. Dhar, M.N. Bera, and G.
Adesso, Measuring Quantum Coherence with Entanglement, Phys. Rev. Lett. 115, 020403 (2015).

[20] E. Chitambar, and M.-H. Hsieh, Relating the Resource Theories of Entanglement and Quantum Coherence, Phys. Rev. Lett. 117, 020402 (2016).

[21] S. Camalet, Simple state preparation for contextuality tests with few observables, Phys. Rev. A 94, 022106 (2016).

[22] S.Camalet, Monogamy inequality for entanglement and local contextuality, Phys. Rev. A 95, 062329 (2017).

[23] M. Schlosshauer, Decoherence, the measurement problem, and interpretations of quantum mechanics, Rev. Mod. Phys. 76, 1267 (2004).

[24] S. Camalet, Effectively classically correlated state of a measured system and a bosonic measurement apparatus, Phys. Rev. A 83, 042106 (2011).

[25] A. W. Marshall, I. Olkin, and B.C. Arnold, Inequalities: Theory of Majorization and its Applications, Second edition, Springer Series in Statistics (Springer, New York, 2011).

[26] A.I. Khinchin, Mathematical Foundations of Information Theory (Dover, New York, 1957).

[27] G.M. Bosyk, S. Zozor, F. Holik, M. Portesi and P.W. Lamberti, A family of generalized quantum entropies: definition and properties, Quant. Inf. Proc. 15, 3393 (2016).

[28] See Supplemental Material for the proofs, which includes Refs. 29, 30].

[29] J.-B. Hiriart-Urruty, and C. Lemaréchal, Fundamentals of Convex Analysis (Springer, Berlin, 2001).

[30] K. Fan, On a theorem of Weyl concerning eigenvalues of linear transformations I., Proc. Nat. Acad. Sci. U.S.A. 35, 652 (1949).

[31] P. Rungta, V. Bužek, C.M. Caves, M. Hillery, and G.J. Milburn, Universal state inversion and concurrence in arbitrary dimensions, Phys. Rev. A 64, 042315 (2001).

[32] M. Kuś, and K. Życzkowski, Geometry of entangled states, Phys. Rev. A 63, 032307 (2001).

[33] F. Verstraete, K. Audenaert, and B. De Moor, Maximally entangled mixed states of two qubits, Phys. Rev. A 64, 012316 (2001).

[34] K. Życzkowski, P. Horodecki, A. Sanpera and M. Lewenstein, Volume of the set of separable states, Phys. Rev. A 58, 883 (1998).

[35] G. Vidal and R.F. Werner, Computable measure of entanglement, Phys. Rev. A 65, 032314 (2002).

[36] K. Chen, S. Albeverio, and S.-M. Fei, Entanglement of Formation of Bipartite Quantum States, Phys. Rev. Lett. 95, 210501 (2005).

[37] Y.-C. Ou and H. Fan, Monogamy inequality in terms of negativity for three-qubit states, Phys. Rev. A 75, 062308 (2007).

[38] C.H. Bennett, H. J. Bernstein, S. Popescu, and B. Schumacher, Concentrating partial entanglement by local operations, Phys. Rev. A 53, 2046 (1996).

[39] V. Vedral, and M. B. Plenio, Entanglement measures and purification procedures, Phys. Rev. A 57, 1619 (1998).

[40] Yao Yao, G. H. Dong, Li Ge, Mo Li, and C. P. Sun, Maximal coherence in a generic basis, Phys. Rev. A 94, 062339 (2016).

[41] T. Endo, Quantum Theory of Phase Relaxation, J. Phys. Soc. Jpn. 56, 1684 (1987). 


\section{Supplemental Material}

In this supplemental material, we show that, for any quantum resource, there is a convex measure of it (proposition (1), that the function $G$, defined by eq.(4), is Schurconvex and convex (proposition 2), and that there exist states for which the two sides of the monogamy inequality

$$
R_{\boldsymbol{d}}\left(\rho_{\mathrm{A}}\right)+E(\rho) \leq R_{\boldsymbol{d}}^{\text {sup }},
$$

are equal, or infinitely close to each other (proposition 3). We also evaluate $g(x)=c o(h)(2 x+1)$, where $c o(h)$ is the convex hull of $h$, defined, on $\left[1, d^{*}\right]$, by

$$
h(y) \equiv \tilde{E}_{\text {max }}-\sup _{\left\{p_{i}\right\} \in \mathcal{F}(y)} G\left(\sum_{i=1}^{d^{*}} p_{i}|i\rangle\langle i|\right),
$$

with $\{|i\rangle\}_{i=1}^{d^{*}}$ any basis, and $\mathcal{F}(y)$ the set of $\left\{p_{i}\right\}_{i=1}^{d^{*}}$ such that $p_{i} \geq 0, \sum_{i=1}^{d^{*}} p_{i}=1$, and $\sum_{i=1}^{d^{*}} \sqrt{p_{i}}=\sqrt{y}$, when system A consists of two two-level systems, and $\tilde{E}$ is the negativity.

A resource measure $\left\{R_{\boldsymbol{d}}\right\}$ obeys

$$
R_{\boldsymbol{d}^{\prime}}\left(\sum_{q} K_{q} \rho_{\mathrm{A}} K_{q}^{\dagger}\right) \leq R_{\boldsymbol{d}}\left(\rho_{\mathrm{A}}\right),
$$

where $K_{q}$ are the Kraus operators of the considered free operation, which are such that $\sum_{q} K_{q}^{\dagger} K_{q}$ is equal to the identity operator on $\mathcal{H}_{\boldsymbol{d}}$, or

$$
\sum_{q} p_{q} R_{\boldsymbol{d}_{q}}\left(\rho_{\mathrm{A}}^{(q)}\right) \leq R_{\boldsymbol{d}}\left(\rho_{\mathrm{A}}\right)
$$

where $p_{q}=\operatorname{tr}\left(K_{q}^{\dagger} K_{q} \rho_{\mathrm{A}}\right)$ is the probability of outcome $q$, $\rho_{\mathrm{A}}^{(q)}=K_{q} \rho_{\mathrm{A}} K_{q}^{\dagger} / p_{q}$ is the corresponding state, and the sum runs over $q$ such that $p_{q}>0$.

Proposition 1. Consider non-negative functions $R_{\boldsymbol{d}}$ that vanish for free states, and satisfy eq.(2) or eq.(3), with Kraus operators $K_{q}$.

There are non-negative convex functions $R_{\boldsymbol{d}}^{c h}$, independent of $\left\{K_{q}\right\}$, that vanish for free states, obey eq.(2) with $\left\{K_{q}\right\}$, if $\boldsymbol{d}_{q}=\boldsymbol{d}^{\prime}$, and fulfill eq.(3) with $\left\{K_{q}\right\}$, if $\left\{R_{\boldsymbol{d}}\right\}$ does.

Proof. Since $R_{\boldsymbol{d}} \geq 0$, it has a convex hull, which is the maximum of the convex functions not larger than $R_{d}$ [1], and is thus non-negative. We define $R_{d}^{c h}$ as this convex hull. As $0 \leq R_{\boldsymbol{d}}^{c h} \leq R_{\boldsymbol{d}}, R_{\boldsymbol{d}}^{c h}$ vanishes whenever $R_{\boldsymbol{d}}$ does, e.g., for free states.

Assume $R_{\boldsymbol{d}}$ and $R_{\boldsymbol{d}^{\prime}}$ satisfy eq.(2), and define the function $H$ by $H\left(\rho_{\mathrm{A}}\right) \equiv R_{\boldsymbol{d}^{\prime}}^{c h}\left[\Phi\left(\rho_{\mathrm{A}}\right)\right]$, where $\Phi\left(\rho_{\mathrm{A}}\right) \equiv$ $\sum_{q} K_{q} \rho_{\mathrm{A}} K_{q}^{\dagger}$. Due to $R_{\boldsymbol{d}^{\prime}}^{\text {ch }} \leq R_{\boldsymbol{d}^{\prime}}$ and eq.(2), $H$ is not greater than $R_{\boldsymbol{d}}$. For the states $\rho_{\mathrm{A}}^{\prime}, \rho_{\mathrm{A}}^{\prime \prime}$, and $\rho_{\mathrm{A}}=$ $\tau \rho_{\mathrm{A}}^{\prime}+\bar{\tau} \rho_{\mathrm{A}}^{\prime \prime}$, where $\bar{\tau}=1-\tau$, and $\tau \in[0,1]$, one obtains

$$
H\left(\rho_{\mathrm{A}}\right)=R_{\boldsymbol{d}^{\prime}}^{c h}\left[\tau \Phi\left(\rho_{\mathrm{A}}^{\prime}\right)+\bar{\tau} \Phi\left(\rho_{\mathrm{A}}^{\prime \prime}\right)\right] \leq \tau H\left(\rho_{\mathrm{A}}^{\prime}\right)+\bar{\tau} H\left(\rho_{\mathrm{A}}^{\prime \prime}\right),
$$

using the linearity of $\Phi$, and the convexity of $R_{\boldsymbol{d}^{\prime}}^{c h}$. Since $H$ is convex and not larger than $R_{\boldsymbol{d}}, H \leq R_{\boldsymbol{d}}^{c h}$, i.e., $R_{\boldsymbol{d}}^{c h}$ and $R_{\boldsymbol{d}^{\prime}}^{c h}$ obey eq.(2).

Assume now that $\left\{R_{d}\right\}$ fulfills eq.(3), and define the functions $I_{\boldsymbol{d}}$ by $I_{\boldsymbol{d}}(\omega) \equiv p R_{\boldsymbol{d}}^{c h}(\omega / p)$, where $\omega$ is any positive Hermitian operator on $\mathcal{H}_{\boldsymbol{d}}$, of trace $p=\operatorname{tr} \omega>0$, and $I_{d}(0) \equiv 0$. For $\omega^{\prime}, \omega^{\prime \prime}$, and $\omega=\tau \omega^{\prime}+\bar{\tau} \omega^{\prime \prime}$, where $\bar{\tau}=1-\tau$, and $\tau \in[0,1]$, the convexity of $R_{d}^{c h}$ leads to

$$
I_{\boldsymbol{d}}(\omega)=p R_{\boldsymbol{d}}^{c h}\left(\tau \frac{p^{\prime}}{p} \frac{\omega^{\prime}}{p^{\prime}}+\bar{\tau} \frac{p^{\prime \prime}}{p} \frac{\omega^{\prime \prime}}{p^{\prime \prime}}\right) \leq \tau I_{\boldsymbol{d}}\left(\omega^{\prime}\right)+\bar{\tau} I_{\boldsymbol{d}}\left(\omega^{\prime \prime}\right),
$$

where $p=\operatorname{tr} \omega$, and $p^{\prime}$ and $p^{\prime \prime}$ are given by similar expressions. Consequently, the function $J$, defined by $J\left(\rho_{\mathrm{A}}\right) \equiv \sum_{q} I_{\boldsymbol{d}_{q}}\left(K_{q} \rho_{\mathrm{A}} K_{q}^{\dagger}\right)$, is convex. Moreover, due to $R_{\boldsymbol{d}_{q}}^{c h} \leq R_{\boldsymbol{d}_{q}}$ and eq.(3),$J$ is not greater than $R_{\boldsymbol{d}}$. Thus, $J \leq R_{\boldsymbol{d}}^{c h}$, i.e., $\left\{R_{\boldsymbol{d}}^{c h}\right\}$ obeys eq.(3). For $\boldsymbol{d}_{q}=\boldsymbol{d}^{\prime}$, since $R_{\boldsymbol{d}^{\prime}}^{\text {ch }}$ is convex, $R_{\boldsymbol{d}^{\prime}}^{c h}\left[\Phi\left(\rho_{\mathrm{A}}\right)\right] \leq J\left(\rho_{\mathrm{A}}\right)$, and hence $R_{\boldsymbol{d}}^{c h}$ and $R_{\boldsymbol{d}^{\prime}}^{c h}$ fulfill eq.(2).

From an arbitrary convex function $R_{\boldsymbol{d}}$, of the density operators on $\mathcal{H}_{\boldsymbol{d}}$, non-negative and bounded, we define, for any state $\rho_{\mathrm{A}}$ of any finite system A,

$$
G\left(\rho_{\mathrm{A}}\right) \equiv \sup _{\{|i\rangle\}} R_{\boldsymbol{d}}\left(\sum_{i=1}^{r} \lambda_{i}\left(\rho_{\mathrm{A}}\right)|i\rangle\langle i|\right),
$$

for $r \leq n(\boldsymbol{d})$, and 0 otherwise, where $n(\boldsymbol{d}) \equiv d_{1} d_{2} \ldots$ is the dimension of $\mathcal{H}_{\boldsymbol{d}}, r$ is the rank of $\rho_{\mathrm{A}}$, and $\lambda_{i}(M)$ denotes the eigenvalues of the Hermitian operator $M$, with $\lambda_{i}(M) \geq \lambda_{i+1}(M)$. The supremum is taken over the bases $\{|i\rangle\}$ of $\mathcal{H}_{\boldsymbol{d}}$.

Proposition 2. The function (44) is

i) Schur-convex, i.e., $G\left(\rho_{\mathrm{A}}\right) \leq G\left(\rho_{\mathrm{A}}^{\prime}\right)$ when $\rho_{\mathrm{A}}^{\prime}$ majorizes $\rho_{\mathrm{A}}$, where $\rho_{\mathrm{A}}$ and $\rho_{\mathrm{A}}^{\prime}$ are states of any finite systems,

ii) convex, i.e., $G\left(\tau \rho_{\mathrm{A}}+\bar{\tau} \rho_{\mathrm{A}}^{\prime}\right) \leq \tau G\left(\rho_{\mathrm{A}}\right)+\bar{\tau} G\left(\rho_{\mathrm{A}}^{\prime}\right)$, where $\rho_{\mathrm{A}}$ and $\rho_{\mathrm{A}}^{\prime}$ are states of a same system, $\bar{\tau}=1-\tau$, and $\tau \in[0,1]$.

Proof. Here, we denote $n(\boldsymbol{d})$ by $n$. For a density operator $\rho_{\mathrm{A}}$ of rank $r \leq n$, we rewrite eq.(4) as

$$
G\left(\rho_{\mathrm{A}}\right)=f\left[\boldsymbol{\lambda}_{n}\left(\rho_{\mathrm{A}}\right)\right],
$$

where the $n$-component vector $\boldsymbol{\lambda}_{n}\left(\rho_{\mathrm{A}}\right)$ is made up of the $r$ nonvanishing eigenvalues $\lambda_{i}\left(\rho_{\mathrm{A}}\right)$, in decreasing order, followed by $n-r$ zeros. The function $f$ of the $n$ component probability vectors $\boldsymbol{p}$, i.e., such that $p_{i} \geq 0$ and $\sum_{i=1}^{n} p_{i}=1$, is given by

$$
f(\boldsymbol{p}) \equiv \sup _{\{|i\rangle\}} R_{\boldsymbol{d}}\left(\sum_{i=1}^{n} p_{i}|i\rangle\langle i|\right),
$$

where the supremum is taken over the bases $\{|i\rangle\}$ of $\mathcal{H}_{\boldsymbol{d}}$. It is clear, from its definition, that $f$ is a symmetric function of the components $p_{i}$. Consider the probability vectors $\boldsymbol{p}, \boldsymbol{p}^{\prime}$, and $\boldsymbol{p}^{\prime \prime} \equiv \tau \boldsymbol{p}+\bar{\tau} \boldsymbol{p}^{\prime}$, where $\bar{\tau}=1-\tau$, 
and $\tau \in[0,1]$. The convexity of $R_{\boldsymbol{d}}$ and the definition (6), give, for any basis $\{|i\rangle\}$ of $\mathcal{H}_{\boldsymbol{d}}, R_{\boldsymbol{d}}\left(\sum_{i=1}^{n} p_{i}^{\prime \prime}|i\rangle\langle i|\right) \leq$ $\tau f(\boldsymbol{p})+\bar{\tau} f\left(\boldsymbol{p}^{\prime}\right)$, which leads to the convexity of $f$. Being symmetric and convex, $f$ is Schur-convex [2].

i) Consider two density operators $\rho_{\mathrm{A}}$ and $\rho_{\mathrm{A}}^{\prime}$, of ranks $r$ and $r^{\prime}$, respectively, such that $\rho_{\mathrm{A}}^{\prime}$ majorizes $\rho_{\mathrm{A}}$. Thus, $r^{\prime} \leq r$. If $r>n$, the inequality $G\left(\rho_{\mathrm{A}}\right) \leq G\left(\rho_{\mathrm{A}}^{\prime}\right)$ is trivially obeyed. If $r \leq n, G\left(\rho_{\mathrm{A}}\right)$ and $G\left(\rho_{\mathrm{A}}^{\prime}\right)$ are both given by eq.(5). Since $\boldsymbol{\lambda}_{n}\left(\rho_{\mathrm{A}}^{\prime}\right)$ majorizes $\boldsymbol{\lambda}_{n}\left(\rho_{\mathrm{A}}\right)$, and $f$ is Schurconvex, $G\left(\rho_{\mathrm{A}}\right) \leq G\left(\rho_{\mathrm{A}}^{\prime}\right)$.

ii) Consider the states $\rho_{\mathrm{A}}$ and $\rho_{\mathrm{A}}^{\prime}$, of a same system, of ranks $r$ and $r^{\prime}$, respectively, and $\rho_{\mathrm{A}}^{\prime \prime} \equiv \tau \rho_{\mathrm{A}}+\bar{\tau} \rho_{\mathrm{A}}^{\prime}$, where $\bar{\tau}=1-\tau$, and $\tau \in] 0,1[$. We assume, without loss of generality, that $r^{\prime} \leq r$. Due to Ky Fan eigenvalue inequality, $\tau \boldsymbol{\lambda}\left(\rho_{\mathrm{A}}\right)+\bar{\tau} \boldsymbol{\lambda}\left(\rho_{\mathrm{A}}^{\prime}\right)$ majorizes $\boldsymbol{\lambda}\left(\rho_{\mathrm{A}}^{\prime \prime}\right)$, where $\boldsymbol{\lambda}(M)$ is the vector made up of the eigenvalues $\lambda_{i}(M)$, in decreasing order [2, 3]. The rank $r^{\prime \prime}$ of $\rho_{\mathrm{A}}^{\prime \prime}$, is hence not smaller than $r$. The convexity inequality for $G$, is obviously satisfied with $\rho_{\mathrm{A}}$ and $\rho_{\mathrm{A}}^{\prime}$, if $r^{\prime \prime}>n$. In the case $r^{\prime \prime} \leq n$, $G\left(\rho_{\mathrm{A}}\right), G\left(\rho_{\mathrm{A}}^{\prime}\right)$, and $G\left(\rho_{\mathrm{A}}^{\prime \prime}\right)$ are given by eq. (15). Moreover, $\tau \boldsymbol{p}+\bar{\tau} \boldsymbol{p}^{\prime}$ majorizes $\boldsymbol{p}^{\prime \prime}$, where $\boldsymbol{p}=\boldsymbol{\lambda}_{n}\left(\rho_{\mathrm{A}}\right)$, and $\boldsymbol{p}^{\prime}$ and $\boldsymbol{p}^{\prime \prime}$ are given by similar expressions, which leads to

$$
f\left(\boldsymbol{p}^{\prime \prime}\right) \leq f\left(\tau \boldsymbol{p}+\bar{\tau} \boldsymbol{p}^{\prime}\right) \leq \tau f(\boldsymbol{p})+\bar{\tau} f\left(\boldsymbol{p}^{\prime}\right),
$$

since $f$ is Schur-convex and convex.

Proposition 3. Consider a system A whose Hilbert space is $\mathcal{H}_{\boldsymbol{d}}$, a $m$-level system $\mathrm{B}, r$ probabilities $p_{i}$, such that $\sum_{i=1}^{r} p_{i}=1$, and $p_{i} \geq p_{i+1}$, with $r \leq m, n(\boldsymbol{d})$, and an entanglement monotone $E$, related to $R_{\boldsymbol{d}}$, by

$$
R_{\boldsymbol{d}}^{\text {sup }}-G\left(\rho_{\mathrm{A}}\right)=\max _{\rho: \operatorname{tr}_{\mathrm{B}} \rho=\rho_{\mathrm{A}}} E(\rho) .
$$

For any $\epsilon>0$, there are pure states $\rho$ of $\mathrm{A}$ and B, such that $\lambda_{i}\left(\rho_{\mathrm{A}}\right)=p_{i}$ for $i \leq r$, and 0 otherwise, and $R_{\boldsymbol{d}}^{\text {sup }}-R_{\boldsymbol{d}}\left(\rho_{\mathrm{A}}\right)-E(\rho)<\epsilon$.

Proof. Consider any pure state $\tilde{\rho}=|\psi\rangle\langle\psi|$ of A and B, where $|\psi\rangle$ has Schmidt coefficients $\sqrt{p_{i}}$, and define $\mathcal{F}$ the set of all pure states $\rho=U \tilde{\rho} U^{\dagger}$, where $U$ is any unitary operator of A. For any $\rho \in \mathcal{F}, E(\rho)=E(\tilde{\rho})$, and $\lambda_{i}\left(\rho_{\mathrm{A}}\right)=p_{i}$ for $i \leq r$, and 0 otherwise. Since $\tilde{\rho}$ is pure, $E(\tilde{\rho})=R_{\boldsymbol{d}}^{\text {sup }}-\sup _{U} R_{\boldsymbol{d}}\left(\operatorname{tr}_{B} U \tilde{\rho} U^{\dagger}\right)$, where the supremum is taken over the unitary operators $U$ of A. Thus, $\sup _{\rho \in \mathcal{F}}\left[E(\rho)+R_{\boldsymbol{d}}\left(\rho_{\mathrm{A}}\right)\right]=R_{\boldsymbol{d}}^{\text {sup }}$, which finishes the proof.

When system A consists of two two-level systems, and $\tilde{E}$ is the negativity, $G\left(\rho_{\mathrm{A}}\right)=\max \left\{0, H\left(\rho_{\mathrm{A}}\right)\right\} / 2$, where

$$
H\left(\rho_{\mathrm{A}}\right)=\sqrt{\left(p_{1}-p_{3}\right)^{2}+\left(p_{2}-p_{4}\right)^{2}}-p_{2}-p_{4},
$$

with $p_{i}=\lambda_{i}\left(\rho_{\mathrm{A}}\right)$ [4], and hence $\tilde{E}_{\max }=1 / 2$. For $d^{*}=2$, in eq.(1), the set $\mathcal{F}(y)$ has only one element $\left\{p_{1}, p_{2}\right\}$. Consequently, $g$ is given by

$$
g(x)=\left(3 / 2-\sqrt{1-2 x^{2}}-\sqrt{1 / 4-x^{2}}\right) / 2,
$$

which increases from 0 to $(3-\sqrt{2}) / 4$, as $x$ varies from 0 to $1 / 2$. For $d^{*}=3$, to evaluate $h(y)$, we first determine the maximum of $H\left(\rho_{\mathrm{A}}\right)$, for $\left\{p_{1}, p_{2}, p_{3}\right\} \in \mathcal{F}(y)$. It is reached for $p_{2}=p_{3}$, and decreases from 1 to 0 , as $y$ varies from 1 to 3 . It leads to

$$
g(x)=1 / 2-k(x)\left(\sqrt{1+[9 / k(x)-3]^{2}}-1\right) / 18,
$$

where $k(x)=(\sqrt{2 x+1}-\sqrt{1-x})^{2}$. This function increases from 0 to $1 / 2$, as $x$ varies from 0 to 1 . For $d^{*}=4$, the maximum of $H\left(\rho_{\mathrm{A}}\right)$, is reached for $p_{2}=p_{3}=p_{4}$, and is equal to $f(y) \equiv(\sqrt{y}+\sqrt{12-3 y})^{2} / 8-1$. For $y \leq 2+\sqrt{3}$, it is positive, and hence $h(y)=1 / 2-f(y) / 2$. For $y \geq 2+\sqrt{3}$, it is negative, and hence $h(y)=1 / 2$. The convex hull $c o(h)$ is equal to $h$, for $y \leq y_{0}$, and to $a(y-4)+1 / 2$, for $y \geq y_{0}$, where $y_{0}$ and $a$ are determined by $h^{\prime}\left(y_{0}\right)=a$, and $h\left(y_{0}\right)=a\left(y_{0}-4\right)+1 / 2$, with $h^{\prime}$ the derivative of $h$. These conditions give $y_{0}=3$ and $a=1 / 4$, and hence

$$
\begin{aligned}
g(x) & =1-(\sqrt{1+2 x}+\sqrt{9-6 x})^{2} / 16 \text { for } x \in[0,1] \\
& =x / 2-1 / 4 \text { for } x \in[1,3 / 2]
\end{aligned}
$$

which increases from 0 to $1 / 2$, as $x$ varies from 0 to $3 / 2$.

[1] J.-B. Hiriart-Urruty, and C. Lemaréchal, Fundamentals of Convex Analysis (Springer, Berlin, 2001).

[2] A. W. Marshall, I. Olkin, and B.C. Arnold, Inequalities: Theory of Majorization and its Applications, Second edition, Springer Series in Statistics (Springer, New York, 2011).

[3] K. Fan, On a theorem of Weyl concerning eigenvalues of linear transformations I., Proc. Nat. Acad. Sci. U.S.A. 35, 652 (1949).

[4] F. Verstraete, K. Audenaert, and B. De Moor, Maximally entangled mixed states of two qubits, Phys. Rev. A 64, 012316 (2001). 